\title{
DEPÓSITO E PERDAS DE CALDA EM SISTEMA DE PULVERIZAÇÃO COM TURBOATOMIZADOR EM VIDEIRA ${ }^{1}$
}

\author{
MARCELO G. BALAN ${ }^{2}$, OTAVIO J. G. ABI SAAB ${ }^{3}$, CRISTHIANE G. SILVA ${ }^{4}$
}

\begin{abstract}
RESUMO: Grande parte dos viticultores da região Norte do Paraná pratica a condução da videira em caramanchão, com mais de 50 aplicações fitossanitárias em um único ciclo. O objetivo deste trabalho foi avaliar essas aplicações com turboatomizador assistido de ar no sistema de pulverização, por meio do depósito de calda nas folhas e perdas. O trabalho foi desenvolvido utilizando-se de cinco condições de aplicação, variando-se tamanho de gotas e volume de aplicação, com quatro repetições, em blocos casualizados. A avaliação do sistema foi feita com a aplicação de uma calda de cloreto de potássio (5\%), sendo o depósito nas folhas medido pela condutividade elétrica, e as perdas, pela comparação entre volumes aplicado e recuperado nas folhas. Para todas as condições, as perdas foram superiores a 48\%. Os maiores volumes aplicados apresentaram as maiores deposições, e gotas maiores apresentaram maior depósito e mesma perda em relação a gotas menores. Os menores volumes não diferiram com relação à deposição, destacando-se as condições gotas maiores a volume baixo e a testemunha utilizada pelo viticultor, que apresentaram as menores perdas. Os resultados demonstram que o turboatomizador é uma importante ferramenta para maximizar as operações de pulverização em uva, e as alterações na configuração das pontas de pulverização devem ser mais estudadas.
\end{abstract}

PALAVRAS-CHAVE: produtos fitossanitários, deposição, jato transportado.

\section{DEPOSITION AND SPRAY LOSSES IN AN AIR-ASSISTED SPRAYER SYSTEM IN GRAPEVINE}

\begin{abstract}
Winegrowers in the north of Paraná State carry on grapevines in arbour and pesticide applications could overcome more than fifty times in a cycle. The aim of this study was to evaluate the features of the air assisted sprayer application system by spray volume deposition and losses. Five treatments and four randomized blocks were used. The treatments varied the droplet size and application volume. The evaluation of the system was done with a $5 \%$ spray solution containing $\mathrm{KCl}$ being the leaves spray deposit measured through electric conductivity and the losses by the comparison among applied and recovered spray volumes on the leaves. For all of the conditions, the losses were above $48 \%$, the conditions of larger volumes presented the largest depositions and larger droplets presented larger deposit and same loss in relation to smaller droplets. The smallest volumes did not differ regarding the deposition standing out the larger droplets to low volume conditions and the witness used by the winegrower (small droplets and low volume), that presented the smallest losses. The results demonstrate that the air-assisted sprayer is an important tool to maximize the pulverization operations in grape and the alterations of nozzles configuration should be better studied.
\end{abstract}

KEYWORDS: agrochemicals, deposition, air-assistance.

\footnotetext{
${ }^{1}$ Parte da dissertação de mestrado do primeiro autor.

${ }^{2}$ Eng $^{\mathrm{o}}$ Agrônomo, Doutorando no Programa de Pós-graduação em Agronomia, Universidade Estadual de Londrina, UEL, Londrina PR, Fone: (0XX43) 3327.3387, balan@uel.br, Bolsista do CNPq.

${ }^{3}$ Eng $^{\mathrm{O}}$ Agrônomo, Prof. Doutor, Departamento de Agronomia, UEL, Londrina - PR.

${ }^{4}$ Discente do curso de Agronomia, UEL, Londrina - PR.

Recebido pelo Conselho Editorial em: 6-10-2005

Aprovado pelo Conselho Editorial em: 10-5-2006
} 


\section{INTRODUÇÃO}

Disseminada em todas as regiões do Brasil, as uvas finas de mesa têm grande importância econômica. Devido às condições climáticas, essas cultivares têm nas doenças fúngicas grande entrave para os resultados econômicos satisfatórios devido à necessidade de um programa intensivo de controle químico, sendo prática comum o uso de produtos fitossanitários preventivos às doenças, podendo alcançar o número, na cultivar Itália, de 40 a 50 aplicações anuais, conforme MURAYAMA (1980). Ainda hoje essa realidade permanece e, em algumas safras, atingem-se até 70 aplicações.

Na região norte do Paraná, o sistema de condução mais difundido é a latada (caramanchão), sendo esse tipo de estrutura capaz de proporcionar grande expansão vegetativa, contribuindo para o aumento da produtividade, facilitando os tratos culturais e fitossanitários, a colheita e a mecanização (GENTA, 2000).

Embora a aquisição de pulverizadores de jato de ar transportado tratorizados (turboatomizadores) por parte dos viticultores não seja comum devido ao seu elevado custo, a região norte do Paraná já possui número expressivo desses equipamentos em substituição aos sistemas estacionários tradicionais, sem assistência de ar junto à barra de pulverização.

HOLOWNICKI et al. (2000) afirmam que os pulverizadores assistidos de ar são os de uso mais comum em frutíferas, sendo fáceis de se operar e efetivos para o controle de pragas e doenças. Entretanto, esses autores apontam que a pulverização em frutíferas é tida como um processo ineficiente, pois mais da metade das doses dos produtos fitossanitários aplicados é perdida.

VEREECKE et al. (2000) destacam que a velocidade de ar gerada pelos ventiladores dos pulverizadores assistidos de ar é um fator importante que influencia na distribuição do líquido aplicado. Com volume muito baixo, ou baixa velocidade do ar produzida pelo ventilador, o produto aplicado alcançará o objetivo irregularmente. Com velocidade muito alta do ar, o produto é soprado através e acima dos alvos. As comparações são necessárias para que surjam melhorias na segurança e efetividade dos métodos de aplicação de produtos fitossanitários nas plantas (CROSS et al., 1997).

A busca em se testar a eficácia desses produtos diretamente em experimentos de campo é corrente. Todavia, experimentos desse tipo são demorados, trabalhosos e ainda sujeitos à ampla gama de variáveis não-controláveis, como incidência de pragas e doenças, oscilações climáticas e variabilidade biológica, sendo difícil a obtenção de diferenças entre os tratamentos. ROCAMORA et al. (2000), estudando o desempenho de pulverizadores com assistência a ar, afirmaram que a redução de volume conduz à diminuição na deposição, mas essa redução de depósito não é proporcional à redução de volume, podendo ser compensada com ajuste de dosagens.

CROSS et al. (1997) consideram que, para a avaliação da efetividade dos métodos de aplicação, a quantificação dos depósitos de pulverização é um dos meios mais rápidos e menos exigentes de recursos. São exemplos da utilização dessa metodologia os trabalhos de SALYANI \& WHITNEY (1988), PERMIN et al. (1992) e DERKSEN \& GRAY (1995).

Quanto ao volume de calda aplicado, STEDEN (1992) cita que, para aplicações de fungicidas em videiras, foram obtidos bons resultados para aplicações com volumes mais baixos (150 a $500 \mathrm{~L} \mathrm{ha}^{-1}$ ); volumes maiores proporcionaram grande ocorrência de perdas por escorrimento nas diversas partes da planta e que, no caso da superfície inferior das folhas, tanto o volume de calda como o tamanho das gotas podem ceder importância a outros fatores, como o jato transportado. SALYANI \& WHITNEY (1988) obtiveram maiores depósitos nas folhas com o aumento do volume de calda aplicada; entretanto, a variação do depósito não ocorreu na mesma proporção da variação do volume. ABI SAAB (2000) concluiu que a utilização de jato transportado melhorou significativamente a cobertura na superfície inferior das folhas, mesmo com redução de $25 \%$ do volume de calda, mas não alterou os valores de deposição e perdas quando comparado à aplicação sem jato transportado. Concluiu, ainda, que, sem o uso de jato transportado, há maior influência do 
volume da calda quando comparado ao tamanho de gotas, na deposição de calda nas folhas. PERGHER et al. (1995), em dois experimentos, observaram que o aumento do volume de aplicação com turboatomizador, na fase que antecede o florescimento, resultou em maiores perdas e menor deposição de calda na folhagem. Para taxa de aplicação baixa (313 a $319 \mathrm{~L} \mathrm{ha}^{-1}$ ), as perdas para o solo foram de 34,5 a 36,8\% e, para taxa de aplicação média (648 a $782 \mathrm{~L} \mathrm{ha}^{-1}$ ) foram de 41,3 a 48,9\%. Esses autores apontam a correlação entre o depósito de calda e o índice de área foliar, em que as taxas de recuperação de calda aplicada, no segundo experimento (desenvolvimento vegetativo pleno), foram maiores. Também PERGHER et al. (1997), estudando a deposição e perdas de produtos fitossanitários em três tipos de pulverizadores com assistência de ar em vinhedos cultivados com condução do tipo espaldeira, concluíram não ter havido diferença significativa no total de perdas entre os três pulverizadores testados. Observaram, também, que as diferentes técnicas de aplicação são capazes de depositar mais de $64 \%$ do volume de calda aplicada nas folhas e frutos. Nesse estudo, o turboatomizador a alto volume proporcionou a melhor uniformidade de distribuição de calda na folhagem. Demonstram, ainda, que o depósito nas folhas tende a aumentar quando o índice de área foliar aumenta.

Uma excelente forma alternativa de avaliar o depósito de calda vem sendo utilizada nos últimos anos, tomando como base a determinação da condutividade elétrica de soluções como parâmetro de avaliação. Esse conceito foi utilizado por ABI SAAB \& ANTUNIASSI (1997) e FOX et al. (1998). Trata-se de um processo com grande versatilidade e precisão para a finalidade de quantificação da calda depositada, em função da condutividade elétrica da solução de lavagem de alvos, conforme as características do sistema apresentado por VELINI et al. (1995). Permite, também, em muitos casos, a utilização da folha para a amostragem, conforme exemplificado nos trabalhos de ABI SAAB (1996; 2000). A possibilidade do uso da própria folha é muito importante, pois é consenso que nenhum alvo artificial é comparável à própria folha quando o alvo é ela mesma.

A determinação das perdas ou do produto não depositado no alvo pretendido também é uma avaliação importante dos sistemas de pulverização, sendo comumente utilizada. Os trabalhos apresentados por PERMIN et al. (1992) e ABI SAAB (2000) podem ser citados como exemplos.

É comum e adequada a associação entre a avaliação da deposição com a avaliação das perdas. Os trabalhos de ABI SAAB (1996; 2000) e HOLOWNICKI et al. (2000) são exemplos dessas avaliações conjuntas.

O objetivo deste trabalho foi avaliar os efeitos do tamanho da gota e do volume de aplicação na deposição e perdas da calda, em sistema de aplicação de produtos fitossanitários com turboatomizadores utilizados pelos viticultores, na região de Marialva - PR.

\section{MATERIAL E MÉTODOS}

O trabalho foi desenvolvido no dia quatro de fevereiro de 2004, em propriedade situada no município de Marialva - PR ( $23^{\circ} 25^{\prime} 32^{\prime}$ 's e $51^{\circ} 48^{\prime} 42^{\prime}$ 'W), com altitude aproximada de $600 \mathrm{~m}$. A classificação climática, segundo Köeppen, é Cfa.

A cultivar de videira utilizada foi a Rubi, com aproximadamente cinco anos de idade, implantada em espaçamento de três metros entre linhas e seis metros entre plantas desencontradas. A forma de condução utilizada é em latada, com altura aproximada de $1,75 \mathrm{~m}$. O parreiral em questão não havia sido podado e ainda restavam alguns cachos de uva dispostos de forma esparsa, e a disposição das folhas constituía-se em apenas um estrato de folhas. Tal fato permitiu melhor condução do trabalho sem a preocupação de eventuais danos, como o da retirada de folhagem para a avaliação da deposição e da cobertura.

O conjunto de pulverização tratorizado utilizado foi de um trator da marca Yanmar 104-DT, 4x4-H, e pulverizador turboatomizador KO A-500, sendo composto por tanque de $500 \mathrm{~L}$ em fibra de vidro, visor de nível com escala graduada, agitador mecânico com hélice de três pás, bomba de três pistões horizontais, com vazão de $45 \mathrm{~L} \mathrm{~min}^{-1}$ a $540 \mathrm{rpm}$, camisa de cerâmica, pressão de 703 a 
$2.110 \mathrm{kPa}$, acionada pela tomada de potência do trator, manômetro, mangueiras e engates para 14 bicos com posição (ângulo) regulável. As pontas de pulverização utilizadas foram JA-1 e JA-2 (jato cônico vazio), gerando gotas menores e 110-LD-015 (jato plano), gerando gotas maiores, marca Jacto.

Para a realização deste trabalho, foram utilizados os seguintes materiais e equipamentos: galões plásticos $(50 \mathrm{~L})$ para preparo da calda, sacos de plástico e papel, papel hidrossensível, corante fluorescente, cloreto de potássio, água destilada e deionizada, trena, réguas plásticas, arame, termoigroanemômetro, luz negra (UV), calculadoras, balanças e vidrarias em geral. Utilizou-se neste trabalho, para a avaliação da deposição e das perdas, o método de determinação da condutividade elétrica das soluções obtidas após a lavagem das folhas de videira (alvos). Para a avaliação da deposição, o método para a obtenção da calda utilizada foi similar ao empregado por ABI SAAB (1996; 2000), sendo composto da mistura de cloreto de potássio (granulado para adubação) em água, nas seguintes proporções: $100 \mathrm{~L}$ de água e $5.000 \mathrm{~g}$ de cloreto de potássio. Para a compreensão do comportamento dos tratamentos utilizados, fez-se a avaliação da deposição e da perda da calda aplicada na cultura.

A definição dos tratamentos foi baseada nas variações possíveis, a partir daquela praticada pelo produtor, que representa a grande maioria dos usuários dessa tecnologia de aplicação na região de Marialva - PR (Figura 1). O experimento foi desenvolvido com cinco tratamentos e quatro repetições, totalizando 20 parcelas de $18 \mathrm{~m}^{2}(3 \times 6 \mathrm{~m})$, no delineamento em blocos ao acaso. Após as aplicações, os valores médios de volume de calda $\left(\mathrm{L} \mathrm{ha}^{-1}\right)$, em cada tratamento, calculados em função da vazão e do tempo médio para o tratamento de $36 \mathrm{~m}^{2}$ em uma só passada, que corresponderam ao tratamento de cada parcela $(6 \mathrm{~m})$ e área de escape, foram determinados e estão descritos na Tabela 1.

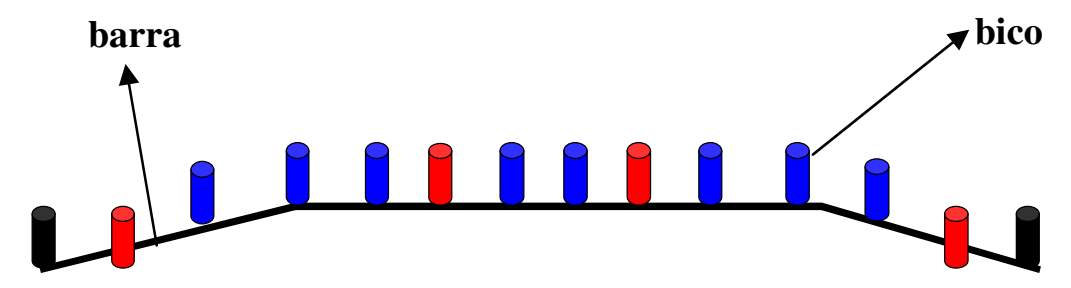

FIGURA 1. Configuração utilizada pelo viticultor (em preto, pontas de pulverização JA-2; em azul, JA-1; em vermelho, pontas fechadas).

TABELA 1. Descrição dos tratamentos em função do tipo de bico e dos valores médios de pressão de trabalho, vazão, tempo de aplicação nas parcelas, volume de calda e DMV.

\begin{tabular}{cccccccc}
\hline Tratamento & Tipo de Ponta & $\begin{array}{c}\text { Número } \\
\text { de Pontas }\end{array}$ & $\begin{array}{c}\text { Pressão } \\
\mathrm{kPa}\end{array}$ & $\begin{array}{c}\text { Vazão** } \\
\left(\mathrm{L} \mathrm{min}^{-1}\right)\end{array}$ & $\begin{array}{c}\text { Tempo Médio } \\
(\mathrm{seg} / 6 \mathrm{~m})\end{array}$ & $\begin{array}{c}\text { Volume } \\
\text { Médio de } \\
\text { Calda } \\
\left(\mathrm{L} \mathrm{ha}^{-1}\right)\end{array}$ & $\begin{array}{c}\text { DMV } \\
(\mu \mathrm{m}) * * *\end{array}$ \\
\hline 1 & 110-LD-015 & 14 & 302 & 0,62 & 10 & 406 & 220 \\
2 & 110-LD-015 & 14 & 302 & 0,62 & 42 & 1.675 & 220 \\
3 & JA-1 & 14 & 1.406 & 0,62 & 10 & 401 & 61 \\
4 & JA-1 & 14 & 1.406 & 0,62 & 42 & 1.674 & 61 \\
$5^{*}$ & JA-2 e JA-1 & 10 & 1.406 & 1,24 e 0,62 & 10 & 358 & 79 e 61 \\
\hline
\end{tabular}

* A configuração desse tratamento é a utilizada pelo viticultor e pode ser observada na Figura 1. ** Vazão por ponta. ***Dados fornecidos pelo fabricante.

As aplicações foram executadas sempre pelo produtor com a intenção de manter o mínimo possível de variações na qualidade das pulverizações. Em cada parcela, foram coletados os dados relativos à temperatura e umidade relativa do ar. Tomou-se o cuidado de efetuar a ordem de aplicação nas parcelas de forma contrária à predominância do vento e coletas de dados logo após a secagem da calda em cada aplicação. Antes de cada aplicação, a vazão de calda foi aferida, 
tomando-se como base o manômetro do equipamento e rotação do motor (540 rpm na tomada de potência), mantendo-se o conjunto ativo fora da área de cada parcela.

Para a avaliação das perdas e do depósito volumétrico da calda sobre as folhas, utilizou-se do método baseado na determinação da condutividade elétrica da calda pulverizada, sugerida inicialmente por VELINI et al. (1995), utilizada e descrita em detalhes por ABI SAAB (1996; 2000) e ABI SAAB \& ANTUNIASSI (1997).

Para a estimativa de perdas e do depósito de calda nas folhas, utilizou-se da eq.(1), proposta por ABI SAAB (1996), que correlaciona a área foliar com o resultado da multiplicação do comprimento pela largura da folha:

$$
\text { Af }=0,920897 \text { Cf Lf }\left(r^{2}=0,97\right)
$$

em que,

Af - área estimada da folha $\left(\mathrm{cm}^{2}\right)$;

Cf - comprimento da folha, medido da inserção do pecíolo até a extremidade do limbo foliar, $\mathrm{cm}, \mathrm{e}$

Lf - largura máxima da folha, medida entre as extremidades laterais do limbo foliar, $\mathrm{cm}$.

Para a estimativa do depósito de calda nas folhas, foi utilizada a fórmula proposta e metodicamente determinada por ABI SAAB (1996), eq.(2), que correlaciona volume de calda depositado nas folhas, condutividade da solução de lavagem e área foliar.

$$
\mathrm{Dtf}=0,401644 \times \mathrm{x} \text { Cs }-0,07619 \text { x Af }-3,51945\left(\mathrm{r}^{2}=0,9940\right)
$$

em que,

Dtf - depósito total de calda estimado sobre a folha, $\mu \mathrm{L}$;

Cs - condutividade elétrica da solução de lavagem da folha, $\mu \mathrm{S} \mathrm{cm}^{-1}$, e

Af - área estimada da folha, $\mathrm{cm}^{2}$.

Após 20 minutos da aplicação de cada tratamento, tempo suficiente para a secagem da calda, foram coletadas 30 folhas representativas, ao acaso, de cada parcela, sendo imediatamente colocadas em sacos plásticos com identificação. Essas folhas foram submetidas aos processos de lavagem, determinação da condutividade elétrica das soluções obtidas e a estimativa da área foliar.

As determinações da condutividade elétrica foram efetivadas para cada dez folhas por saco plástico. Para cada conjunto, saco plástico e folhas, foram acrescidos $300 \mathrm{~mL}$ de água destilada deionizada. Cada conjunto foi agitado por aproximadamente 30 segundos e, imediatamente após, foi tomada a medida de condutividade da solução obtida. A seguir, foram determinados o comprimento e a largura de cada folha para a estimativa de área.

Os dados de condutividade foram aplicados à eq.(2), fornecendo valores estimados do volume total de calda depositado em cada grupo de dez folhas.

Os valores obtidos na eq.(2) foram aplicados à eq.(3), fornecendo o depósito estimado de calda por $\mathrm{cm}^{2}$ de folha. Para cada aplicação, foi calculada a média do volume de calda nas 30 folhas amostradas. em que,

$$
\mathrm{Vcf}=\mathrm{Dtf} / \mathrm{Af},
$$

Vcf - volume de calda depositado sobre a folha, $\mu \mathrm{L} \mathrm{cm}^{-2}$;

Dtf - depósito total de calda estimado sobre a folha, $\mu \mathrm{L}$, e

Af - área estimada das dez folhas, $\mathrm{cm}^{2}$.

Em cada tratamento avaliado, a estimativa das perdas (volume não depositado nas folhas) foi realizada por meio da comparação entre o volume de calda recuperado nas folhas e o volume efetivamente aplicado durante as pulverizações. Para a determinação do volume recuperado, 
realizou-se avaliação da área foliar correspondente a $1 \mathrm{~m}^{2}$ de solo na cultura (índice de área foliar). Assim, multiplicando-se o depósito médio pela área foliar, obteve-se o total de depósito correspondente a $1 \mathrm{~m}^{2}$ de solo. Comparando-se esse valor com o volume de calda efetivamente aplicado, estimaram-se as perdas de calda.

Para a estimativa do índice de área foliar, coletaram-se todas as folhas de três parcelas de $1 \mathrm{~m}^{2}$. Utilizando-se da eq.(1) para a estimativa da área de cada folha, obteve-se a estimativa do índice de área foliar médio da parreira de valor 1,05, característico do final de ciclo produtivo da parreira estudada.

As análises estatísticas foram feitas por meio da análise de variância, complementada com o teste de Tukey para comparação de médias, com probabilidade de erro de 5\%.

\section{RESULTADOS E DISCUSSÃO}

Os resultados relativos ao depósito de calda nas folhas e às perdas estão apresentados na Tabela 2.

TABELA 2. Análise de variância, com respectivas médias do depósito de calda recuperado das folhas $\left(\mu \mathrm{L} \mathrm{cm}^{-2}\right)$ e da percentagem de perdas da calda.

\begin{tabular}{ccccc}
\hline Tratamentos & $\begin{array}{c}\text { Volume } \\
\left(\mathrm{L} \mathrm{ha}^{-1}\right)\end{array}$ & $\begin{array}{c}\text { Tamanho de } \\
\text { Gotas }\end{array}$ & $\begin{array}{c}\text { Depósito de Calda } \\
\left(\mu \mathrm{cm}^{-2}\right)\end{array}$ & Perdas (\%) \\
\hline 1 & 406 & GG & $1,98 \mathrm{c}$ & $48,7 \mathrm{c}$ \\
2 & 1.675 & GG & $6,32 \mathrm{a}$ & $60,4 \mathrm{ab}$ \\
3 & 401 & GP & $1,38 \mathrm{c}$ & $63,9 \mathrm{ab}$ \\
4 & 1.674 & GP & $5,29 \mathrm{~b}$ & $66,9 \mathrm{a}$ \\
5 & 358 & GP & $1,54 \mathrm{c}$ & $54,9 \mathrm{bc}$ \\
\hline F Tratamentos & & & $135,41 * *$ & $9,35 * *$ \\
F Blocos & & $1,31 \mathrm{n} . \mathrm{s}$. & $1,35 \mathrm{n} . \mathrm{s}$. \\
C.V. $(\%)$ & & 12,11 & 5,99 \\
\hline
\end{tabular}

Médias seguidas de mesma letra não diferem entre si, pelo teste de Tukey, a $5 \%$ de probabilidade. n.s. indica que não houve significância. ** indica significância a $1 \%$ de probabilidade.

Nos trabalhos de SALYANI \& WHITNEY (1988) e PERGHER et al. (1997), os maiores volumes de calda aplicados resultaram em maior depósito sobre as folhas. No presente trabalho, tal constatação foi verificada onde os maiores volumes (tratamentos 2 e 4), independentemente do tamanho das gotas, apresentaram maiores deposições quando comparados aos demais tratamentos. ABI SAAB (2000), sem o uso do jato transportado, não observou influência do tamanho de gotas no depósito de calda nas folhas. Possivelmente, o auxílio de ar propiciou melhor condição para maior depósito na aplicação de gotas maiores, menos sujeitas à deriva, o que promoveu o maior depósito para o tratamento 2, quando comparado ao tratamento 4 (gotas menores).

O esperado é que a perda em alto volume seja maior, quando comparada a baixo volume, conforme afirmam SALYANI \& CROMWELL (1993). No entanto, no presente trabalho, isso só ocorreu para as gotas grandes. Esse fato pode indicar que também há relação entre o tamanho de gotas. O tratamento 3, com baixo volume, não diferiu significativamente dos tratamentos de maiores volumes ( 2 e 4 ). As gotas menores no tratamento 3 parecem ser a causa de maiores perdas, possivelmente em função da deriva.

Embora sem diferença estatística, é possível observar a tendência esperada de que, para um mesmo volume aplicado e depósitos diferentes, proporciona-se menor índice de perda para o tratamento de maior depósito, podendo ser observado nos tratamentos de maior volume (2 e 4) e nos de menor volume, em que as gotas maiores apresentaram maior deposição (tratamento 1), seguida da testemunha (tratamento 5) e gotas menores (tratamento 3 ). 
Sobre as afirmações de SALYANI \& WHITNEY (1988) e ROCAMORA et al. (2000), de que a relação entre volume de calda aplicado e deposição não serem proporcionais, os resultados mostram que também pode haver influência do tamanho da gota. No presente trabalho, observou-se que a desproporcionalidade pode ser mais evidente para as gotas maiores do que para as menores. Esse resultado pode estar associado aos fatos de que as gotas menores estão mais sujeitas aos efeitos do vento, devido a sua menor massa, e que as gotas maiores podem provocar maiores perdas por escorrimento.

Observou-se que, para volume menor aplicado e depósitos iguais, a percentagem de perdas foi significativamente menor para o tratamento com o uso de gotas grandes (tratamento 1), seguido do tratamento 5, com gotas pequenas distribuídas pela configuração utilizada pelo produtor. Essas perdas foram menores que aquelas observadas por ABI SAAB (2000) que, sem o uso do jato transportado, obteve como menor percentagem de perda o valor de 64,5\%. Essa constatação, provavelmente, está ligada ao auxílio de ar que favorece o alcance do alvo pelas gotas.

A modificação na distribuição e na vazão das pontas utilizadas na barra de pulverização pode ser vista como uma possibilidade de favorecer a deposição e reduzir as perdas. Como para a cultura da uva, os produtos fitossanitários são aplicados levando-se em consideração a concentração por volume aplicado e não dose por unidade de área, a utilização de menores volumes pode proporcionar menores percentagens de perdas, uso de menores quantidades de produtos fitossanitários, economia de água, maior rendimento operacional e menor contaminação do produto final e meio ambiente, concordando com o observado por ABI SAAB (2000). Entretanto, essas modificações devem ser feitas criteriosamente para evitar a diminuição significativa da deposição do produto fitossanitário, como ocorrido neste trabalho, em função da grande variação do volume aplicado.

\section{CONCLUSÕES}

O sistema de gota grande e volume de aplicação maior proporcionou a maior deposição e, nos sistemas de gota grande e volume de aplicação menor e testemunha, não houve diferença de deposição em relação ao tamanho das gotas; entretanto, nesse caso, as gotas maiores promoveram menores perdas.

A utilização de gotas maiores, no maior volume de aplicação, aumentou a deposição sem aumentar as perdas. No menor volume de aplicação, houve diminuição de perdas sem haver, entretanto, aumento de deposição.

A variação da deposição em relação ao volume aplicado pode ser influenciada pelo tamanho da gota.

\section{AGRADECIMENTOS}

Agradecimento especial à Associação Norte Paranaense de Estudos em Fruticultura ANPEF, que possibilitou a execução deste trabalho.

\section{REFERÊNCIAS}

ABI SAAB, O.J.G. Avaliação de um sistema de aplicação de defensivos utilizado em videiras no Município de Londrina - PR. 1996. 65 f. Dissertação (Mestrado em Energia na Agricultura) Faculdade de Ciências Agronômicas, Universidade Estadual Paulista, Botucatu, 1996.

ABI SAAB, O.J.G. Avaliação de cobertura e depósitos de agrotóxicos em videiras com o uso de diferentes técnicas de aplicação e condições operacionais. 2000. $84 \mathrm{f}$. Tese (Doutorado em Energia na Agricultura) - Faculdade de Ciências Agronômicas, Universidade Estadual Paulista, Botucatu, 2000.

ABI SAAB, O.J.G.; ANTUNIASSI, U.R. Avaliação do depósito de calda em um sistema de aplicação de defensivos utilizados em videiras. Energia na Agricultura, Botucatu, v.12, n.2, p.1-11, 1997. 
CROSS, J.V.; MURRAY, R.A.; RIDOUT, M.S.; WALKLATE, P.J. Quantification of spray deposits and their variability on apple trees. In: ASPECTS OF APPLIED BIOLOGY, 48., 1997, Long Ashton. Proceedings... Warwick: Association of Applied Biologists, 1997. p.217-24.

DERKSEN, R.C.; GRAY, R.L. Deposition and air speed patterns of air-carrier apple orchard sprayer. Transitions of the ASAE, St. Joseph,v.38, n.1, p.5-11, 1995.

FOX, R.D.; DERKSEN, R.C.; BRAZEE, R.D.; OZKAN, H.E.; DOWNER, R.A. The reached spray deposit analyzer, a tool for measuring spray deposit by measuring conductivity. In: INTERNATIONAL CONFERENCE ON AGRICULTURAL ENGINEERING, 1998, Oslo. Paper... Oslo, 1998. 7 p. (Paper 98-A-008).

GENTA, W. A cultura da videira. Marialva: PLANTA, 2000. 19 p.

HOLOWNICKI, R.; DORUCHOWSKI, G.; SWIECHOWSKI, W.; GODYN, A. Automatic self adjusting air-jet sprayer concept for fruit trees. In: INTERNATIONAL CONFERENCE ON AGRICULTURAL ENGINEERING, 2000, Warwick. Paper... Warwick, 2000. 10 p. (Paper 00PM-053).

MURAYAMA, S. Fruticultura. 2.ed. Campinas: Instituto Campineiro de Ensino Agrícola, 1980. $385 \mathrm{p}$.

PERGHER, G.; GUBIANI, R.; TONNETO, G. The effect of spray application rate and airflow rate on foliar deposition in a hedgerow vineyard. Journal of Agricultural Engineering Research, London, v.61, n.1, p.205-16, 1995.

PERGHER, G.; GUBIANI, R; TONNETO, G. Foliar deposition and pesticide losses from three air-assisted sprayers in a hedgerow vineyard. Crop Protection, Oxford, v.16, n.1, p.25-33, 1997.

PERMIN, O.; JØRGERSEN, L.N.; PERSSON, K. Biological effect of herbicides and fungicides, deposition and drift hazards, when using different sizes of hydraulic flat fan nozzle for the application. Tidsskrift for planteavl, Lyngby, v.96, n.5, p.531-42, 1992.

ROCAMORA, M.C.; VAL, L.; PÉREZ, M. Performance of air-assisted spraying on horticultural crops. In: INTERNATIONAL CONFERENCE ON AGRICULTURAL ENGINEERING, 2000, Warwick. Paper... Warwick, 2000. 8 p. (Paper 00-PM-028)

SALYANI, M.; CROMWELL, R.P. Spray drift from ground and aerial applications. Transactions of the ASAE, St. Joseph, v.35, n.4, p.1113-220, 1993.

SALYANI, M.; WHITNEY, J.D. Evaluation of methodologies for field studies of spray deposition. Transactions oh the ASAE, St. Joseph, v.31, n.2, p.390-5, 1988.

STEDEN, C. Untersuchungen zum einflu $\beta$ der tropfengröße auf die belagsbildung und die biologische wirksamkeit gegen Oidium tuckeri Berk. an reben. 1992. $118 \mathrm{f}$. Inaugural Dissertation zur Erlangung des Doktorgrades Justus - Universität Gießen, Justus - Liebig, 1992.

VELINI, E.D.; ANTUNIASSI, U.R.; MARTINS, D.; TRINDADE, M.L.B.; SILVA, M.S. Utilização da condutividade elétrica para avaliação do depósito da calda de pulverização em alvos ou folhas. In.: CONGRESSO BRASILEIRO DA CIÊNCIA DAS PLANTAS DANINHAS, 20., 1995, Florianópolis. Resumos... Florianópolis: Sociedade Brasileira da Ciência das Plantas Daninhas, 1995. p 427.

VEREECKE, E.; LANGENAKENS, J.; DE MORR, A.; PIETERS, M.; JAEKEN, P. The air distribution generated by air-assisted sprayers. In: INTERNATIONAL CONFERENCE ON AGRICULTURAL ENGINEERING, 2000, Warwick. Paper... Warwick, 2000. 10 p. (Paper 00PM-071) 